\title{
The ratio of CD8+ lymphocytes to tumor-infiltrating suppressive FOXP3+ effector regulatory T cells is associated with treatment response in invasive breast cancer
}

Takayuki Kadoya ( $\nabla$ takayukikadoya@gmail.com )

Hiroshima University

\section{Noriko Goda}

Hiroshima University

Shinsuke Sasada

Hiroshima University

Hideo Shigematsu

National Hospital Organization Kure Medical Center and Chugoku Cancer Center

Norio Masumoto

Hiroshima University

Koji Arihiro

Hiroshima University

Hiroyoshi Nishikawa

National Cancer Center

Shimon Sakaguchi

Osaka University

Morihito Okada

Hiroshima University

\section{Research Article}

Keywords: Breast cancer, regulatory T cell, tumor-infiltrating lymphocyte, FOXP3, CD8, neoadjuvant chemotherapy

Posted Date: January 27th, 2022

DOI: https://doi.org/10.21203/rs.3.rs-1287664/v1

License: (c) (i) This work is licensed under a Creative Commons Attribution 4.0 International License. Read Full License 


\section{Abstract}

Purpose: FOXP3+ tumor-infiltrating lymphocytes (TILs) are prototypical immunosuppressive TILs in breast cancer. However, the FOXP3+ TIL subset of effector regulatory T cells (eTregs) cannot be detected with conventional immunohistochemical staining. Therefore, we evaluate the immunosuppressive potential of eTregs and the role of immunostimulatory CD8+ TILs in invasive breast cancer.

Methods: Fresh TILs were extracted from 84 patients with breast cancer and divided into $\mathrm{CD}^{+} \mathrm{FOXP}^{+}$regulatory $\mathrm{T}$ cells (Tregs) and CD8+ cytotoxic T lymphocytes (CTLs) by flow cytometry. Tregs were further classified into eTregs (CD4 ${ }^{+}$FOXP3 ${ }^{\text {high }}{ }^{2}$ 45RA ${ }^{-}$), other FOXP3 ${ }^{+}$Treg subsets (naïve and non-Tregs), and total CD $8^{+}$CD $4^{-}$TILs.). The association between TILs subpopulation, clinicopathological characteristics, and the response to chemotherapy was evaluated.

Results: The median eTreg proportion among total CD4+ TILs was 18.7\% (interquartile range [IQR], 16.4-25.5\%), whereas this proportion for CD8 ${ }^{+}$TILs was $124 \%$ (IQR, 87.5-140\%). The proportion of eTregs to total FOXP3 ${ }^{+}$TILs varied widely among patients (median, 65.6\%; range, 10.1-93.2\%). In an immunosuppression assay with Treg subsets, only eTregs displayed potent immunosuppression. Among the patients who received neoadjuvant chemotherapy (NAC), the pathological complete response(pCR) rate was significantly higher among individuals with a high $\mathrm{CD}^{+} / \mathrm{eTreg}$ ratio $(90.2 \%$ vs. $33.3 \%)$. TILs predominance and CD8/eTreg ratio were independent predictive factors for pCR to chemotherapy $(P=0.043$ and $P=$ 0.034 , respectively).

Conclusions: The CD8+/eTreg ratio is a simple, optimal indicator of cancer immunity, and a high CD8+/eTreg ratio may enhance the prognosis and treatment response of patients with invasive breast cancer. Further studies are warranted to validate the present findings.

\section{Introduction}

Breast cancer is a common malignancy that leads to morbidity and mortality in women worldwide[1-3]. According to the Global Cancer Statistics report in 2021, approximately 2.2 million women were diagnosed with breast cancer with nearly 700,000 deaths[2].

Several studies have shown that tumor-infiltrating lymphocytes (TILs) in breast cancer are strongly associated with treatment response and patient prognosis [4-7]. TILs are typically quantitatively evaluated using hematoxylin-eosin (HE)stained samples. Subsequently, qualitative assessments are performed as TILs are functionally heterogeneous and consist of immunoprogressive or immunosuppressive components [8]. Regulatory T cells (Tregs), the most representative immunosuppressive TILs, are positive for transcription factor forkhead box P3 (FOXP3) and regulate anticancer immunity [9-11]. Some studies have reported increased Tregs in breast cancer as an adverse prognostic factor [12-15]. In contrast, other studies have suggested that Tregs predict favorable outcomes[16-19]. Thus, the role of Tregs in breast cancer TILs remains controversial [20]. Meanwhile, CD8+ TILs are representative immunoprogressive TILs and have been confirmed as an independent predictive factor for treatment response [22,23] or survival [24]. Furthermore, several studies recommend the evaluation of the CD8+/FOXP3+ ratio of TILs as sensitive markers of tumor immune responses in breast cancer rather than evaluation of FOXP3+or CD8+ TILs alone [25-29]. These studies indicate that the balance of TIL components, which have conflicting functions, influence the prognosis of breast cancer.

FOXP3, detected by immunohistochemical staining, is a general Treg marker. However, FOXP3+ cells are functionally heterogeneous and can be classified into three fractions using flow cytometry based on the expression levels of FOXP3 and naïve T cell marker CD45RA: naïve Treg, effector Treg (eTreg), and non-Treg. Only eTreg cells have a suppressive function, and the other fractions are nonsuppressive and secrete inflammatory cytokines [10,30]. In colorectal cancer, it has been reported that the variation of tumor-infiltrating Treg component is caused by immunologically relevant genes and that these variations affect disease prognosis [31]. However, infiltration of only eTregs from total FOXP3+ cells cannot be detected 
using FOXP3 immunohistochemical staining. Moreover, the variation in eTreg infiltration has not been investigated in breast cancer, and the role of eTregs in the disease remains unclear. Herein, we examined the association between tumor-infiltrating eTregs and CD8+TILs and the clinical outcomes of patients with invasive breast cancer.

\section{Materials And Methods Patients and treatments}

In total, 84 patients with early breast cancer who underwent complete resection between December 2015 and November 2016 were included. Patients with noninvasive diseases were excluded from the study. The $\mathrm{N}$-acetylcysteine (NAC) regimen consisted of four cycles of docetaxel $(75 \mathrm{mg} / \mathrm{m} 2$, every three weeks), followed by four cycles of FEC (500 mg/m2 5fluorouracil, $100 \mathrm{mg} / \mathrm{m} 2$ epirubicin, and $500 \mathrm{mg} / \mathrm{m} 2$ cyclophosphamide; every three weeks). Patients with human epidermal growth factor receptor 2 (HER2)-positive breast cancer received trastuzumab ( $8 \mathrm{mg} / \mathrm{m} 2$ for the first dose and $6 \mathrm{mg} / \mathrm{m} 2$ thereafter) every three weeks together with docetaxel. Pathological compete response (pCR) was defined as the absence of invasive residuals in the primary lesion and axillary lymph nodes[32]. The protocol of this study was approved by the Ethics Committee of Hiroshima University and was conducted in accordance with the Declaration of Helsinki. Written informed consent was obtained from all patients.

\section{Breast cancer tissue collection and extraction of TILs}

Fresh samples of invasive breast cancer tissues were collected via core needle biopsy, vacuum-assisted biopsy (Mammotome Elite; Mammotome, Cincinnati, OH, USA), or surgery. Biopsy specimens from patients who had received NAC were collected before treatment. Tumor samples were collected as follows: three to five samples using 16-gauge biopsy needles, $>6$ samples using 13-gauge Mammotome needles, or an area of at least $10 \mathrm{~mm} \times 10 \mathrm{~mm} \times 2 \mathrm{~mm}$ shaved using a razor during surgery. Fresh TILs were extracted as described previously[31]. Fresh tissues were rapidly cut into small pieces using tissue scissors and homogenized using a GentleMACS dissociator (Miltenyi Biotech, Bergisch Gladbach, Germany), and the TILs were collected from the cell suspensions.

\section{Flow cytometry}

Fresh TILs were washed with phosphate-buffered saline containing $2 \%$ fetal calf serum and allophycocyanin-conjugated anti-CD4 mAb (BD Biosciences, Franklin Lakes, NJ, USA), V500-conjugated anti-CD8 mAb (BD Biosciences), fluorescein isothiocyanate-conjugated anti-CD45RA mAb (BD Biosciences), and Fixable Viability dye (eBioscience, San Diego, CA, USA). Intracellular staining of FOXP3 was performed using an anti-FOXP3 mAb and FOXP3 Staining Buffer Set (eBioscience) according to the manufacturer's instructions. The cells were analyzed using LSRFortessa (BD Biosciences) and FlowJo software (Tree Star, Ashland, OR, U

\section{Determination of TILs subpopulations}

TILs were categorized according to the methods described in a previous report $[8,28]$. TILs were gated into CD4-CD8+ T cells and CD4+CD8 $\mathrm{T}$ cells, and the CD4+CD8 $\mathrm{T}$ cell fraction was further gated based on FOXP3 and CD45RA expression as

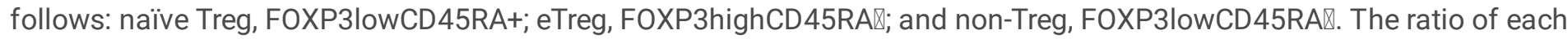
TIL subpopulation to the total CD4+CD8 TILs was measured. Lymphocytes were collected from peripheral blood mononuclear cells (PBMCs), and lymphocytes from normal breast tissue (LNBT) were used as a control for validation.

\section{Treg suppression assay}

Naïve Treg, eTreg, and non-Treg cells were isolated from TILs, LNBT, and PBMCs using FACS Aria II (BD Biosciences). Responder CD25-CD4+ T cells (control) were purified from PBMCs, labeled with carboxyfluorescein succinimidyl ester (CFSE), and mixed with purified components at a ratio of 1 responder T cell:3 cells of each component. Treg Suppression Inspector reagent (Miltenyi Biotec) was then added according to the manufacturer's instructions. The cells were cultured for $5 \mathrm{~d}$, and the proliferation of CFSE-labeled cells was evaluated. 


\section{Pathological assessment and evaluation of stromal TILs}

Histological characteristics, such as histology, nuclear grade, estrogen receptor (ER) and HER2 status, Ki-67 labeling index, and stromal TILs, were assessed by two pathologists. ER and HER2 were assessed according to the American Society of Clinical Oncology/College of American Pathologists Guidelines [35]. The Ki-67 labeling index was scored as high ( $\geq 20 \%)$ or low $(<20 \%)$. Stromal TILs were assessed on HE-stained slides of maximum number of tumor lesions using the methodology proposed by the 2014 International TILs Working Group [36]. Lymphocyte-predominant breast cancer (LPBC) was defined as stromal TIL $\geq 60 \%$.

\section{Statistical analyses}

Basic statistics for the TIL subpopulations are expressed as the median and interquartile range (IQR). The Wilcoxon ranksum test was performed for multiple pairwise comparisons. Kaplan-Meier curve analysis was performed for assessment of disease-free survival with the log-rank test. Receiver operating characteristic curves were constructed to determine the cutoff values of parameters predicting $\mathrm{PCR}$. Statistical significance was set at $\mathrm{P}<0.05$. All statistical analyses were performed using JMP Pro14 SAS software (SAS Institute Inc., Cary, NC, USA).

\section{Results}

Table 1 summarizes the clinicopathological characteristics of 84 patients with invasive breast cancer. Among them, 32 patients (32.2\%) had nodal metastasis and 25 (29.8\%) had lymphocyte predominant breast cancer (LPBC). In total, 50 (59.5\%) were ER-positive diseases and 28 (33.3\%) were HER2-positive. A total of 39 patients received NAC, and 20 (51.3\%) achieved pCR. Among the fresh TILs, the median number of total TILs was $6.9 \times 10^{5}\left(\right.$ IQR $\left.1.2-82 \times 10^{5}\right)$ cells, which was considered sufficient for the analyses. $C D 4^{+} C D 8^{-} T$ cells were categorized as eTregs, naïve Tregs, and non-Tregs via flow cytometric analysis for CD45RA and FOXP3 expression (Figure 1a, left). Treg proportions varied among cases (Figure 1a, right). In the suppression assay, although eTregs demonstrated strong immunosuppressive activity, naïve Tregs and nonTregs displayed no immunosuppressive effects (Figure 1b). The proportion of eTregs among total FOXP3 ${ }^{+}$TILs also varied markedly among individuals (median, 65.6\%; range, 10.1-93.2\%) (Figure 1c). Among the CD4 ${ }^{+}$TILs, PBMCs, and LNBTs, the median percentage of total FOXP3 ${ }^{+}$cells was the highest, followed by that of eTregs, non-Tregs, and a low proportion of naïve Tregs; however, the proportion of eTreg cells was higher in the TILs compared with the PBMCs and LNBTs (Figure 1d). The median percentage of $\mathrm{CD} 8^{+}$TILs to CD $4^{+}$TILs was $124 \%$ (IQR, 87.5-140). 


\begin{tabular}{|ll|}
\hline & Number (\%) \\
\hline Age (year), median (range) & $57(33-83)$ \\
\hline T status & $22(26.1)$ \\
\hline T1 & $54(64.2)$ \\
\hline T2 & $4(4.7)$ \\
\hline T3 & $4(4.7)$ \\
\hline T4 & \\
\hline Nodal metastasis & $52(61.9)$ \\
\hline Negative & $32(38.1)$ \\
\hline Positive & \\
\hline Nuclear grade & $4(4.8)$ \\
\hline 1 & $27(32.1)$ \\
\hline 2 & $53(63.1)$ \\
\hline 3 & $31(36.9)$ \\
\hline LVI positive & $50(59.5)$ \\
\hline ER positive & $28(33.3)$ \\
\hline HER2 positive & \\
\hline Ki-67 labeling index & $20(23.8)$ \\
\hline$<20 \%$ & 64 (76.2) \\
\hline$\geq 20 \%$ & $59(70.2)$ \\
\hline Stromal TILs & $25(29.8)$ \\
\hline Non-LPBC & $39(46.4)$ \\
\hline LPBC & $19(48.7)$ \\
\hline Neoadjuvant chemotherapy & $20(51.3)$ \\
\hline Non-pCR & \\
\hline pCR & \\
\hline ER, estrogen receptor; HER2, human epidermal growth factor receptor 2; LPBC, lymphocyte predominant breast cancer; \\
LVI, lymphovascular invasion; pCR, pathological complete response; TILs, tumor-infiltrating lymphocytes.
\end{tabular}

Table 2 shows the association of clinicopathological features with FOXP3 ${ }^{+}$, eTregs, and CD8 ${ }^{+}$TILs. CD8 ${ }^{+}$TILs were significantly associated with HER2 amplification and LPBC. However, the Treg components were similar among different breast cancer subtypes. FOXP3 ${ }^{+}$expression and eTreg proportion were not significantly associated with clinicopathological factors. The therapeutic responses of the 39 patients receiving NAC were evaluated. Receiver operating characteristic curves were constructed to determine the cut-off values of parameters predicting PCR to stratify patients into groups with high or low levels of each parameter (eTregs, $9.1 \% \mathrm{CD}^{+}$cells: high $\mathrm{n}=20$, low $\mathrm{n}=19 ; \mathrm{CD} 8^{+}, 113 \%$ of $\mathrm{CD} 4^{+}$cells: high $\mathrm{n}=18$, low $=$ 
21; $\mathrm{CD}^{+} /$eTreg, 13.3: high $n=13$, low $n=26$; $\mathrm{CD}^{+} / \mathrm{FOXP3}^{+}, 4.6$ : high $n=12$, low $n=27$ ). Patients with TN and HER2 subtypes achieved higher $\mathrm{PCR}$ rates than those with the luminal subtype $(\mathrm{P}<0.05)$. There was no significant difference in $\mathrm{pCR}$ rates between patients with high and low eTregs. Patients displaying a high $\mathrm{CD} 8^{+} / \mathrm{eTreg}$ ratio achieved significantly higher pCR than those displaying a low $C D 8^{+}$/eTreg ratio $(P=0.001)$, whereas $p C R$ rates did not differ significantly according to $\mathrm{CD}^{+} / \mathrm{FOXP3}^{+}$ratio (Figure 2 ). 
Table 2

Relationship between clinicopathological features and TILs subpopulations

\begin{tabular}{|c|c|c|c|c|c|c|c|c|c|c|c|}
\hline & \multicolumn{2}{|l|}{ FOXP3 $^{+}$} & \multicolumn{2}{|l|}{ Fr-I } & \multicolumn{2}{|l|}{ Fr-II } & \multicolumn{2}{|l|}{ Fr-III } & \multicolumn{2}{|l|}{$\mathrm{CD}^{+}$} & \\
\hline & $\begin{array}{l}\text { Median } \\
\text { (IQR) }\end{array}$ & $P$ & $\begin{array}{l}\text { Median } \\
\text { (IQR) }\end{array}$ & $P$ & $\begin{array}{l}\text { Median } \\
\text { (IQR) }\end{array}$ & $P$ & $\begin{array}{l}\text { Median } \\
\text { (IQR) }\end{array}$ & $P$ & $\begin{array}{l}\text { Median } \\
\text { (IQR) }\end{array}$ & $P$ & \\
\hline \multicolumn{12}{|l|}{ Age } \\
\hline$<50$ year & 28 & $\begin{array}{l}31.7 \\
(24.9- \\
38.8)\end{array}$ & \multirow[t]{2}{*}{0.913} & $\begin{array}{l}1.7 \\
(0.8- \\
4.2)\end{array}$ & \multirow[t]{2}{*}{0.970} & $\begin{array}{l}21.2 \\
(15.6- \\
26.5)\end{array}$ & \multirow[t]{2}{*}{0.605} & $\begin{array}{l}6.7 \\
(3.9- \\
10.0)\end{array}$ & \multirow[t]{2}{*}{0.239} & $\begin{array}{l}113.0 \\
(93.3- \\
141.0)\end{array}$ & \multirow[t]{2}{*}{0.849} \\
\hline $\begin{array}{l}\geq 50 \\
\text { year }\end{array}$ & 56 & $\begin{array}{l}30.7 \\
(24.2- \\
40.9)\end{array}$ & & $\begin{array}{l}2.0 \\
(0.9- \\
3.3)\end{array}$ & & $\begin{array}{l}20.1 \\
(16.5- \\
24.9)\end{array}$ & & $\begin{array}{l}7.8 \\
(5.0- \\
13.1)\end{array}$ & & $\begin{array}{l}116.0 \\
(86.0- \\
140.5)\end{array}$ & \\
\hline \multicolumn{12}{|l|}{ T status } \\
\hline $\mathrm{T} 1$ & 22 & $\begin{array}{l}34.8 \\
(25.5- \\
40.3)\end{array}$ & \multirow[t]{2}{*}{0.401} & $\begin{array}{l}1.9 \\
(1.0- \\
4.1)\end{array}$ & \multirow[t]{2}{*}{0.935} & $\begin{array}{l}22.4 \\
(18.0- \\
26.0)\end{array}$ & \multirow[t]{2}{*}{0.344} & $\begin{array}{l}8.3 \\
(5.0- \\
12.7)\end{array}$ & \multirow[t]{2}{*}{0.558} & $\begin{array}{l}113.5 \\
(76.5- \\
153.5)\end{array}$ & \multirow[t]{2}{*}{0.665} \\
\hline $\mathrm{T} 2-4$ & 62 & $\begin{array}{l}30.0 \\
(24.4- \\
40.3)\end{array}$ & & $\begin{array}{l}2.0 \\
(0.9- \\
3.3)\end{array}$ & & $\begin{array}{l}19.9 \\
(16.1- \\
25.5)\end{array}$ & & $\begin{array}{l}7.3 \\
(4.5- \\
11.2)\end{array}$ & & $\begin{array}{l}115.5 \\
(89.5- \\
139.5)\end{array}$ & \\
\hline \multicolumn{12}{|c|}{ Nodal metastasis } \\
\hline Negative & 52 & $\begin{array}{l}30.7 \\
(23.8- \\
38.8)\end{array}$ & \multirow[t]{2}{*}{0.289} & $\begin{array}{l}2.0 \\
(1.0- \\
3.3)\end{array}$ & \multirow[t]{2}{*}{0.971} & $\begin{array}{l}20.0 \\
(15.7- \\
24.9)\end{array}$ & \multirow[t]{2}{*}{0.384} & $\begin{array}{l}7.7 \\
(4.5- \\
11.8)\end{array}$ & \multirow[t]{2}{*}{0.751} & $\begin{array}{l}117.0 \\
(86.0- \\
150.3)\end{array}$ & \multirow[t]{2}{*}{0.522} \\
\hline Positive & 32 & $\begin{array}{l}32.3 \\
(26.0- \\
42.7)\end{array}$ & & $\begin{array}{l}2.0 \\
(0.8- \\
3.8)\end{array}$ & & $\begin{array}{l}21.4 \\
(16.6- \\
28.6)\end{array}$ & & $\begin{array}{l}7.3 \\
(5.0- \\
11.1)\end{array}$ & & $\begin{array}{l}108.5 \\
(88.0- \\
133.0)\end{array}$ & \\
\hline \multicolumn{12}{|c|}{ Nuclear grade } \\
\hline $1-2$ & 31 & $\begin{array}{l}33.8 \\
(24.5- \\
42.6)\end{array}$ & \multirow[t]{2}{*}{0.347} & $\begin{array}{l}2.2 \\
(0.8- \\
4.2)\end{array}$ & \multirow[t]{2}{*}{0.307} & $\begin{array}{l}20.0 \\
(13.0- \\
26.8)\end{array}$ & \multirow[t]{2}{*}{0.792} & $\begin{array}{l}7.3 \\
(5.3- \\
14.3)\end{array}$ & \multirow[t]{2}{*}{0.191} & $\begin{array}{l}113.0 \\
(86.0- \\
128.0)\end{array}$ & \multirow[t]{2}{*}{0.319} \\
\hline 3 & 53 & $\begin{array}{l}29.3 \\
(24.5- \\
38.7)\end{array}$ & & $\begin{array}{l}1.9 \\
(1.0- \\
3.2)\end{array}$ & & $\begin{array}{l}20.3 \\
(16.5- \\
25.4)\end{array}$ & & $\begin{array}{l}7.3 \\
(4.1- \\
10.6)\end{array}$ & & $\begin{array}{l}117.0 \\
(87.0- \\
154.0)\end{array}$ & \\
\hline \multicolumn{12}{|l|}{ LVI } \\
\hline Negative & 57 & $\begin{array}{l}30.7 \\
(24.7- \\
39.4)\end{array}$ & \multirow[t]{2}{*}{0.427} & $\begin{array}{l}2.0 \\
(1.0- \\
3.8)\end{array}$ & \multirow[t]{2}{*}{0.457} & $\begin{array}{l}20.0 \\
(14.8- \\
23.0)\end{array}$ & \multirow[t]{2}{*}{0.193} & $\begin{array}{l}7.1 \\
(4.5- \\
10.0)\end{array}$ & \multirow[t]{2}{*}{0.292} & $\begin{array}{l}117.0 \\
(87.0- \\
147.5)\end{array}$ & 0.515 \\
\hline Positive & 27 & $\begin{array}{l}33.8 \\
(23.7- \\
42.0)\end{array}$ & & $\begin{array}{l}1.8 \\
(0.8- \\
3.2)\end{array}$ & & $\begin{array}{l}22.0 \\
(16.5- \\
29.0)\end{array}$ & & $\begin{array}{l}8.8 \\
(5.5- \\
12.2)\end{array}$ & & $\begin{array}{l}104.0 \\
(86.0- \\
132.0)\end{array}$ & \\
\hline ER & & & & & & & & & & & \\
\hline Negative & 34 & $\begin{array}{l}28.7 \\
(25.7- \\
38.2)\end{array}$ & 0.417 & $\begin{array}{l}1.5 \\
(0.7- \\
2.8)\end{array}$ & 0.084 & $\begin{array}{l}20.3 \\
(16.1- \\
25.8)\end{array}$ & 0.971 & $\begin{array}{l}7.3 \\
(4.8- \\
9.9)\end{array}$ & 0.384 & $\begin{array}{l}114.0 \\
(90.5- \\
153.5)\end{array}$ & 0.444 \\
\hline
\end{tabular}

ER, estrogen receptor; HER2, human epidermal growth factor receptor 2; LPBC, lymphocyte predominant breast cancer; LVI, lymphovascular invasion; TILs, tumor-infiltrating lymphocytes. 


\begin{tabular}{|c|c|c|c|c|c|c|c|c|c|c|c|}
\hline & FOXP3 $^{+}$ & & Fr-I & & Fr-II & & Fr-III & & $\mathrm{CD}^{+}$ & & \\
\hline & $\begin{array}{l}\text { Median } \\
\text { (IQR) }\end{array}$ & $P$ & $\begin{array}{l}\text { Median } \\
\text { (IQR) }\end{array}$ & $P$ & $\begin{array}{l}\text { Median } \\
\text { (IQR) }\end{array}$ & $P$ & $\begin{array}{l}\text { Median } \\
\text { (IQR) }\end{array}$ & $P$ & $\begin{array}{l}\text { Median } \\
\text { (IQR) }\end{array}$ & $P$ & \\
\hline Positive & 50 & $\begin{array}{l}32.6 \\
(23.8- \\
41.9)\end{array}$ & & $\begin{array}{l}2.0 \\
(1.1- \\
4.1)\end{array}$ & & $\begin{array}{l}20.1 \\
(16.2- \\
25.5)\end{array}$ & & $\begin{array}{l}7.5 \\
(4.8- \\
14.2)\end{array}$ & & $\begin{array}{l}115.0 \\
(85.8- \\
138.3)\end{array}$ & \\
\hline \multicolumn{12}{|l|}{ HER2 } \\
\hline Negative & 56 & $\begin{array}{l}32.7 \\
(25.1- \\
40.9)\end{array}$ & \multirow[t]{2}{*}{0.553} & $\begin{array}{l}2.0 \\
(0.9- \\
3.8)\end{array}$ & \multirow[t]{2}{*}{0.296} & $\begin{array}{l}20.2 \\
(14.2- \\
25.4)\end{array}$ & \multirow[t]{2}{*}{0.439} & $\begin{array}{l}7.8 \\
(5.3- \\
14.0)\end{array}$ & \multirow[t]{2}{*}{0.028} & $\begin{array}{l}113.0 \\
(83.8- \\
131.0)\end{array}$ & \multirow[t]{2}{*}{0.031} \\
\hline Positive & 28 & $\begin{array}{l}28.7 \\
(23.2- \\
38.7)\end{array}$ & & $\begin{array}{l}1.9 \\
(0.5- \\
2.7)\end{array}$ & & $\begin{array}{l}20.2 \\
(17.1- \\
26.5)\end{array}$ & & $\begin{array}{l}5.7 \\
(3.5- \\
9.8)\end{array}$ & & $\begin{array}{l}130.5 \\
(93.3- \\
192.8)\end{array}$ & \\
\hline \multicolumn{12}{|c|}{ Ki-67 labeling index } \\
\hline$<20 \%$ & 20 & $\begin{array}{l}33.3 \\
(26.0- \\
40.6)\end{array}$ & \multirow[t]{2}{*}{0.834} & $\begin{array}{l}1.6 \\
(0.5- \\
4.2)\end{array}$ & \multirow[t]{2}{*}{0.436} & $\begin{array}{l}20.2 \\
(13.0- \\
26.1)\end{array}$ & \multirow[t]{2}{*}{0.896} & $\begin{array}{l}7.1 \\
(5.1- \\
15.1)\end{array}$ & \multirow[t]{2}{*}{0.501} & $\begin{array}{l}108.5 \\
(87.5- \\
131.8)\end{array}$ & \multirow[t]{2}{*}{0.386} \\
\hline$\geq 20 \%$ & 64 & $\begin{array}{l}30.7 \\
(24.2- \\
40.1)\end{array}$ & & $\begin{array}{l}2.0 \\
(1.0- \\
3.3)\end{array}$ & & $\begin{array}{l}20.2 \\
(16.5- \\
25.5)\end{array}$ & & $\begin{array}{l}7.5 \\
(4.0- \\
11.3)\end{array}$ & & $\begin{array}{l}117.0 \\
(86.5- \\
150.3)\end{array}$ & \\
\hline \multicolumn{12}{|l|}{$\begin{array}{l}\text { Stromal } \\
\text { TILs }\end{array}$} \\
\hline $\begin{array}{l}\text { Non- } \\
\text { LPBC }\end{array}$ & 25 & $\begin{array}{l}36.7 \\
(25.5- \\
42.4)\end{array}$ & \multirow[t]{2}{*}{0.136} & $\begin{array}{l}1.9 \\
(0.9- \\
2.9)\end{array}$ & \multirow[t]{2}{*}{0.769} & $\begin{array}{l}20.8 \\
(18.8- \\
26.4)\end{array}$ & \multirow[t]{2}{*}{0.178} & $\begin{array}{l}9.8 \\
(5.1- \\
15.4)\end{array}$ & \multirow[t]{2}{*}{0.193} & $\begin{array}{l}155.0 \\
(92.0- \\
213.5)\end{array}$ & \multirow[t]{2}{*}{0.002} \\
\hline LPBC & 59 & $\begin{array}{l}30.6 \\
(24.1- \\
38.3)\end{array}$ & & $\begin{array}{l}2.0 \\
(0.9- \\
3.9)\end{array}$ & & $\begin{array}{l}19.9 \\
(13.0- \\
25.5)\end{array}$ & & $\begin{array}{l}7.3 \\
(4.6- \\
10.0)\end{array}$ & & $\begin{array}{l}110.0 \\
(86.0- \\
128.0)\end{array}$ & \\
\hline
\end{tabular}

ER, estrogen receptor; HER2, human epidermal growth factor receptor 2; LPBC, lymphocyte predominant breast cancer; LVI, lymphovascular invasion; TILs, tumor-infiltrating lymphocytes.

The factors predicting pCR were assessed based on some cut-offs (Table 3). LPBC and high-CD8 tumor were significant predictors for $\mathrm{PCR}(\mathrm{P}=0.006$ and $\mathrm{P}=0.020$, respectively). After investigating the balance of CD8+CTLs and Treg subpopulations, $\mathrm{CD} 8 / \mathrm{e}$ Treg ratio was the most promising parameter for predicting $\mathrm{pCR}$ and the positive predictive value was 91.7\%. In the multivariate analysis, both LPBC and high-CD8/eTreg ratio were independent predictors for pCR (odds ratio [OR] 10.1, P = 0.043 and OR 18.7, $\mathrm{P}=0.034$, respectively) (Table 4). 
Table 3

Pathological response according to tumor subtypes and TILs subpopulation

\begin{tabular}{|c|c|c|c|}
\hline & Non-pCR & PCR & $P$ \\
\hline Overall & $19(48.7)$ & $20(51.3)$ & \\
\hline ER & & & 0.056 \\
\hline Negative & 7 (33.3) & $14(66.7)$ & \\
\hline Positive & $12(66.7)$ & $6(33.3)$ & \\
\hline HER2 & & & 1 \\
\hline Negative & $8(47.1)$ & $9(52.9)$ & \\
\hline Positive & $11(50.0)$ & $11(50.0)$ & \\
\hline Ki-67 labeling index & & & 0.407 \\
\hline$<20 \%$ & $4(66.7)$ & $2(33.3)$ & \\
\hline$\geq 20 \%$ & $15(45.5)$ & $18(54.5)$ & \\
\hline Stromal TILs & & & 0.006 \\
\hline Non-LPBC & $17(65.4)$ & $9(34.6)$ & \\
\hline LPBC & $2(15.4)$ & $11(84.6)$ & \\
\hline \multicolumn{4}{|l|}{$<$ TILs subpopulation> } \\
\hline FOXP3 & & & 0.32 \\
\hline Low & $11(42.3)$ & 15 (57.7) & \\
\hline High & $8(61.5)$ & $5(38.5)$ & \\
\hline eTreg & & & 0.082 \\
\hline Low & $11(39.3)$ & $17(60.7)$ & \\
\hline High & $8(72.7)$ & $3(27.3)$ & \\
\hline CD8 & & & 0.02 \\
\hline Low & $18(60.0)$ & $12(40.0)$ & \\
\hline High & $1(11.1)$ & $8(88.9)$ & \\
\hline CD8/FOXP3 & & & 0.007 \\
\hline Low & $16(66.7)$ & $8(33.3)$ & \\
\hline High & $3(20.0)$ & $12(80.0)$ & \\
\hline CD8/eTreg ratio & & & 0.001 \\
\hline Low & $18(66.7)$ & $9(33.3)$ & \\
\hline High & $1(8.3)$ & $11(91.7)$ & \\
\hline
\end{tabular}


Table 4

Logistic regression analysis for predicting pathological complete response

\begin{tabular}{|lllll|}
\hline & Univariate analysis & & \multicolumn{2}{l|}{ Multivariate analysis } \\
\hline & OR $(95 \% \mathrm{Cl})$ & $P$ & OR $(95 \% \mathrm{Cl})$ & $P$ \\
\hline ER-positive & $0.25(0.07-0.95)$ & 0.042 & $0.17(0.02-1.19)$ & 0.074 \\
\hline HER2-positive & $0.89(0.25-3.16)$ & 0.855 & $0.32(0.04-2.44)$ & 0.274 \\
\hline Ki-67 labeling index $\geq 20 \%$ & $2.40(0.39-15.0)$ & 0.349 & $1.19(0.11-12.6)$ & 0.884 \\
\hline LPBC & $10.4(1.88-57.4)$ & 0.007 & $10.1(1.08-94.2)$ & 0.043 \\
\hline CD8/eTreg ratio high & $22.0(2.44-198)$ & 0.006 & $18.7(1.25-279)$ & 0.034 \\
\hline $\begin{array}{l}\text { Cl, confidence interval; ER, estrogen receptor; HER2, human epidermal growth factor receptor 2; LPBC, lymphocyte } \\
\text { predominant breast cancer; OR, odds ratio. }\end{array}$ & & & \\
\hline
\end{tabular}

\section{Discussion}

In this study, we focused on the immunological functional heterogeneity of TILs and evaluated the balance of immunoprogressive and immunosuppressive power of TILs in patients with breast cancer. To the best of our knowledge, this study is the first to assess eTregs in breast cancer TILs and verify the association between the functional balance of TILs and clinical outcome of breast cancer. We demonstrated the functional heterogeneity of FOXP3+ Tregs using fresh TIL samples. Tregs are components of CD4+ T cells and essential effector cells that maintain immune homeostasis $[9,10,37]$. Although Tregs are considered to be homogeneously immunosuppressive TILs in breast cancer, we demonstrated that only eTregs had an immunosuppressive effect. We found that eTreg cells were more abundant in breast cancer tissues than in PBMCs and LNBTs, which is consistent with previous reports on other cancer types, such as colorectal [31]and gastric cancer[38]. The difference in the infiltration of Treg subpopulations indicates that T cells are activated and acquire functions in the tumor microenvironment. It is worth noting that patients with low levels of eTreg to total FOXP3+TILs can overestimate their immunosuppressive function by the FOXP3 immunohistochemical staining method. Thus, these patients may lead to conflicting results in some studies that are based on immunohistochemical evaluation of FOXP3 for breast cancer prognosis. LPBC is considered as a strong biomarker for pathological response to NAC regardless of molecular subtypes [4,6] and survival in triple-negative and HER2+ subtype[5]. Our findings indicate that the CD8+/eTreg ratio is a more promising predictor of $\mathrm{pCR}$ and progression-free survival compared to the CD8+/FOXP3+ ratio. We only showed the likelihood of better prognosis of the CD8+/FOXP3+ ratio, and the low numbers of patients may be attributable to a lack of power to show significant difference. Moreover, approximately $90 \%$ of patients were in the early stage. It has been reported that CD15s antibodies can specifically identify eTreg cells [40]. The dual immunohistochemical staining of FOXP3 and CD15s may be a promising method for detecting eTreg infiltration with a large sample size.

Despite the findings of this study, it has several limitations. First, the number of cases was small, and the follow-up period was short. Our findings cannot elucidate the mechanism underlying increased eTregs in the tumor microenvironment. Moreover, we did not evaluate the other TIL components. Recently, an exhaustive and promising evaluation of breast cancer TILs by single-cell RNA sequencing has been reported[41]. The genomic background or mechanism that causes these variations in TIL components will be elucidated in the near future. In addition, eTregs express immune checkpoint molecules, such as cytotoxic T-lymphocyte-associated protein 4 (CTLA-4) and programmed cell death-1 (PD-1), suggesting that controlling tumor-infiltrating Tregs may be a potential target for cancer immunotherapy [42-45]. Our findings may be linked to other studies evaluating the response to immune checkpoint inhibitors in patients with breast cancer to build on their significance. 
In summary, our study indicates that the functional heterogeneity of FOXP3+ TILs represents specific variations in the immunological balance in invasive breast cancer. A high CD8/eTreg ratio enhances treatment response in invasive patients with breast cancer. Further studies are warranted to validate these findings.

\section{Conclusion}

The CD8+/eTreg ratio is a simple and optimal marker for cancer immunity and an increase in this ratio suggests an enhanced prognosis and treatment response in patients with invasive breast cancer; however, further large-scale studies are warranted to validate the present findings.

\section{Abbreviations}

CFSE: Carboxyfluorescein succinimidyl ester

ER: Estrogen receptor

LPBC: Lymphocyte predominant breast cancer

OR: Odds ratio

PBMCs: Peripheral blood mononuclear cells

TILs: Tumor-infiltrating lymphocytes

\section{Declarations}

\section{AUTHOR CONTRIBUTIONS}

NG and SH designed the study, and wrote the initial draft of the manuscript. SS contributed to the analysis and interpretation of data and assisted in the preparation of the manuscript. NM, KA, HN, SS,MO and TK contributed to data interpretation and critically reviewed the manuscript. All authors have approved the final version of the manuscript and agree to be accountable for all aspects of the work with respect to accuracy or integrity.

\section{ACKNOWLEDGMENTS}

We thank the members of Department of Pathology, Hiroshima University Hospital for sample collection and Kanako Teshima at Experimental Immunology, WPI Immunology Frontier Research Center, Osaka University for technical guidance and helpful support.

\section{CONFLICTS OF INTEREST}

The authors declare no competing financial interests.

\section{FUNDING}

This study was supported by Grants-in-Aid for Scientific Research (Grant number:15K19854)from the Ministry of Education, Culture, Sports, Science and Technology of Japan. 


\section{DATA AVAILABILITY STATEMENT}

The datasets generated during and/or analysed during the current study are available from the corresponding author on reasonable request.

\section{Conflicts of Interest:}

The authors declare no competing interests.

\section{References}

1. Ghoncheh M, Pournamdar Z, Salehiniya H. Incidence and Mortality and Epidemiology of Breast Cancer in the World. Asian Pac J Cancer Prev. 2016;17:43-6.

2. Savas P, Salgado R, Denkert C, Sotiriou C, Darcy PK, Smyth MJ, Loi S. Clinical relevance of host immunity in breast cancer: from TILs to the clinic. Nat Rev Clin Oncol. 2016;13:228-41.

3. Loi S, Michiels S, Salgado R, Sirtaine N, Jose V, Fumagalli D et al. Tumor-infiltrating lymphocytes are prognostic in triple negative breast cancer and predictive for trastuzumab benefit in early breast cancer: results from the FinHER trial. Ann Oncol. 2014; 25: 1544-50.

4. Denkert C, von Minckwitz G, Darb-Esfahani S, Lederer B, Heppner BI, Weber KE,et al. Tumour-infiltrating lymphocytes and prognosis in different subtypes of breast cancer: a pooled analysis of 3771 patients treated with neoadjuvant therapy. Lancet Oncol. 2018 Jan;19:40-50.

5. Stanton SE, Disis ML. Clinical significance of tumor-infiltrating lymphocytes in breast cancer. J Immunother Cancer. 2016;4:59.

6. Hendry S, Salgado R, Gevaert T, Russell PA, John T, Thapa B et al. Assessing Tumor-infiltrating Lymphocytes in Solid Tumors: A Practical Review for Pathologists and Proposal for a Standardized Method From the International Immunooncology Biomarkers Working Group: Part 1: Assessing the Host Immune Response, TILs in Invasive Breast Carcinoma and Ductal Carcinoma In Situ, Metastatic Tumor Deposits and Areas for Further Research. Adv Anat Pathol. 2017;24:235-51.

7. Sakaguchi S. Reguratory T cell and immune tolerance. Cell.2008; 133: 775-87.

8. Sakaguchi S, Miyara M, Cosantonio CM. FOXP3+ regulatory T cell in the human immune system. Nat Rev Immunol 2010;10: 490-500.

9. Nishikawa H, Sakaguchi S.Regulatory T cells in cancer immunotherapy. Curr Opin Immunol 2010;27: 1-7.

10. Aruga T, Suzuki E, Saji S, Horiguchi S, Horiguchi K, Sekine S et al. A low number of tumor-infiltrating FOXP3-positive cells during primary systemic chemotherapy correlates with favorable anti-tumor response in patients with breast cancer. Oncol Rep. 2009; 22: 273-78.

11. de Kruijf EM, van Nes JG, Sajet A, Tummers QR, Putter H, Osanto Set al. The predictive value of HLA class I tumor cell expression and presence of intratumoral Tregs for chemotherapy in patients with early breast cancer. Clin Cancer Res. 2010; 16: 1272-80.

12. Ladoire S, Arnould L, Apetoh L, Coudert B, Martin F, Chauffert B, Fumoleau P, Ghiringhelli F. Pathologic complete response to neoadjuvant chemotherapy of breast carcinoma is associated with the disappearance of tumor-infiltrating foxp3+ regulatory T cells. Clin Cancer Res. 2008; 14: 2413-20

13. Bates GJ, Fox SB, Han C, Leek RD, Garcia JF, Harris AL, Banham AH. Quantification of regulatory T cells enables the identification of high-risk breast cancer patients and those at risk of late relapse. J Clin Oncol. 2006; 24: 5373-80.

14. Gobert M, Treilleux I, Bendriss-Vermare N, Bachelot T, Goddard-Leon S, Arfi V et al. Regulatory T cells recruited through CCL22/CCR4 are selectively activated in lymphoid infiltrates surrounding primary breast tumors and lead to an adverse

Page $12 / 15$ 
clinical outcome. Cancer Res. 2009; 69: 2000-9.

15. West NR, Kost SE, Martin SD, Milne K, Deleeuw RJ, Nelson BH, Watson PH. Tumour-infiltrating FOXP3(+) lymphocytes are associated with cytotoxic immune responses and good clinical outcome in oestrogen receptor-negative breast cancer. Br J Cancer. 2013; 108: 155-62.

16. Ladoire S, Arnould L, Mignot G, Coudert B, Rébé C, Chalmin F, Vincent J et al. Presence of Foxp3 expression in tumor cells predicts better survival in HER2-overexpressing breast cancer patients treated with neoadjuvant chemotherapy. Breast Cancer Res and Tre. 2011; 125: 65-72.

17. Yeong J, Thike AA, Lim JC, Lee B, Li H, Wong SC,et al. Higher densities of Foxp3+ regulatory T cells are associated with better prognosis in triple-negative breast cancer. Breast Cancer Res and Tre. 2017; 163: 21-35.

18. Mahmoud SM, Paish EC, Powe DG, Macmillan RD, Lee AH, Ellis IO, Green AR. An evaluation of the clinical significance of FOXP3+ infiltrating cells in human breast cancer. Breast Cancer Rese and Tre. 2011; 127: 99-108.

19. deLeeuw RJ, Kost SE, Kakal JA, Nelson BH. The prognostic value of FoxP3+ tumor-infiltrating lymphocytes in cancer: a critical review of the literature. Clin Cancer Res. 2012; 18: 3022-9.

20. Seo AN, Lee HJ, Kim EJ. Kim HJ, Jang MH, Lee HE et al. Tumour-infiltrating CD8+ lymphocytes as an independent predictive factor for pathological complete response to primary systemic therapy in breast cancer. $\mathrm{Br} \mathrm{J}$ Cancer. 2013; 109: 2705-13.

21. Ali HR, Provenzano E, Dawson SJ, Blows FM, Liu B, Shah M et al. Association between CD8+T cell infiltration and breast cancer survival in 12439 patients. Ann Oncol. 2014; 25: 1536-43

22. Adams S, Gray RJ, Demaria S. Prognostic value of tumor-infiltrating lymphocytes in triple-negative breast cancers from two phase III randomized adjuvant breast cancer trials: ECOG 2197 and ECOG 1199. J Clin Oncol. 2014; 32: 2959-66.

23. Liu F, Lang R, Zhao J, Zhang X, Pringle GA, Fan Y et al. CD8\ cytotoxic T cell and FOXP3\ regulatory T cell infiltration in relation to breast cancer survival and molecular subtypes. Breast Cancer Rese and Tre. 2011; 130: 645-55.

24. Miyashita M, Sasano H, Tamaki K. Tumor-infiltrating CD8+ and FOXP3+ lymphocytes in triple-negative breast cancer: its correlation with pathological complete response to neoadjuvant chemotherapy. Breast Cancer Res and Tre. 2014; 148: 525-34.

25. Miyashita M, Sasano H, Tamaki K. Prognostic significance of tumor-infiltrating CD8+ and FOXP3+ lymphocytes in residual tumors and alterations in these parameters after neoadjuvant chemotherapy in triple-negative breast cancer: a retrospective multicenter study. Breast Cancer Res. 2015; 17: 124.

26. Asano Y, Kashiwagi S. Tumour-infiltrating CD8 to FOXP3 lymphocyte ratio in predicting treatment responses to neoadjuvant chemotherapy of aggressive breast cancer. Br J Surg. 2016; 103: 845-54.

27. Semeraro M, Adam J, Stoll G. The ratio of CD8+/FOXP3 T lymphocytes infiltrating breast tissues predicts the relapse of ductal carcinoma in situ. Oncoimmunology. 2016; 5: e1218106.

28. Miyara M, Yoshioka Y, Kitoh A, Shima T, Wing K, Niwa A et al. Functional delineation and differentiation dynamics of human CD4+ T cells expressing the FoxP3 transcription factor. Immunity. 2009; 30: 899-911.

29. Saito T, Nishikawa H, Wada H, Nagano Y, Sugiyama D, Atarashi K et al. Two FOXP3(+)CD4(+) T cell subpopulations distinctly control the prognosis of colorectal cancers. Nat Med. 2016; 22: 679-84.

30. Fumagalli D, Bedard PL, Nahleh Z, Michiels S, Sotiriou C, Loi S et al. A common language in neoadjuvant breast cancer clinical trials: proposals for standard definitions and endpoints. Lancet Oncol. 2012; 13:e240-8.

31. Sugiyama D, Nishikawa H, Maeda Y, Nishioka M, Tanemura A, Katayama I et al. Anti-CCR4 mAb selectively depletes effector-type FoxP3+CD4+ regulatory T cells, evoking antitumor immune responses in humans. Proc Natl Acad Sci U S A. 2013; 110: 17945-50.

32. Hammond ME, Hayes DF, Dowsett M, Allred DC, Hagerty KL, Badve S et al. American Society of Clinical Oncology/College of American Pathologists guideline recommendations for immunohistochemical testing of estrogen and progesterone receptors in breast cancer. J Clin Oncol. 2010; 28: 2784-95. 
33. Bhargava R, Dabbs DJ. Interpretation of human epidermal growth factor receptor 2 (HER2) in situ hybridization assays using 2013 update of American Society of Clinical Oncology/College of American Pathologists HER2 Guidelines. J clin Oncol. 2014; 32: 1855.

34. Salgado R, Denkert C, Demaria S, Sirtaine N, Klauschen F, Pruneri G et al. The evaluation of tumor-infiltrating lymphocytes (TILs) in breast cancer: recommendations by an International TILs Working Group 2014. Ann Oncol. 2015; 26: 259-71.

35. Sakaguchi S, Sakaguchi N, Asano M, Itoh M, Toda M. Immunologic self-tolerance maintained by activated T cells expressing IL-2 receptor alpha-chains (CD25). Breakdown of a single mechanism of self-tolerance causes various autoimmune diseases. J Immunol. 1995;155: 1151-64.

36. Nagase H, Takeoka T, Urakawa S, Morimoto-Okazawa A, Kawashima A, Iwahori K et al. ICOS+ Foxp3+ TILs in gastric cancer are prognostic markers and effector regulatory T cells associated with Helicobacter pylori. Int J Cancer. 2017; 140: 686-95.

37. Loi S, Sirtaine N, Piette F, Salgado R, Viale G, Van Eenoo F et al. Prognostic and predictive value of tumor-infiltrating lymphocytes in a phase III randomized adjuvant breast cancer trial in node-positive breast cancer comparing the addition of docetaxel to doxorubicin with based chemotherapy: BIG 02-98. J Clin Oncol. 2013; 31: 860-7.

38. Miyara M, Chader D, Sage E, Sugiyama D, Nishikawa H, Bouvry D et al. Sialyl Lewis x(CD15s) identifies highly differentiated and most suppressive FOXP3 ${ }^{\text {high }}$ regulatory T cells in humans. Proc Natl Acad Sci U S A. 2015; 112: 722530

39. Savas P, Virassamy B, Ye C, Salim A, Mintoff CP, Caramia F et al. Single-cell profiling of breast cancer T cells reveals a tissue-resident memory subset associated with improved prognosis. Nat Med. 2018 ;24:986-93.

40. Grinberg-Bleyer Y, Oh H, Desrichard A Bhatt DM, Caron R, Chan TA et al. NF-kB c-Rel Is Crucial for the Regulatory T Cell Immune Checkpoint in Cancer. Cell. 2017;170:1096-108

41. Tanaka A, Sakaguchi S. Regulatory T cells in cancer immunotherapy. Cell Res. 2017;27:109-18

42. Speiser DE, Ho PC, Verdeil G. Regulatory circuits of T cell function in cancer. Nat Rev Immunol. 2016;16:599-611.

43. Shitara K, Nishikawa H. Regulatory T cells: a potential target in cancer immunotherapy. Ann N Y Acad Sci. 2018;1417:104-15.

\section{Figures}

\section{Figure 1}

a:TILs subpopulation by flow cytometry in invasive breast cancer.

CD $4^{-} \mathrm{CD} 8^{+} \mathrm{T}$ cells and $\mathrm{CD} 4^{+} \mathrm{CD} 8^{-} \mathrm{T}$ cells in TILs were separated by flow cytometry (left), and the $\mathrm{CD} 4^{+} \mathrm{CD} 8^{-}$cells were further classified into Naiive Treg, eTreg, and non Treg cells by FOXP3 and CD45RA staining.

b:Suppression assay of breast cancer TILs.

eTreg cells demonstrated the strong suppressive activity, whereas Naiive and non Treg cells had no suppressive effects. Arrows and "\%" indicates the proliferation of co-cultured responder T cell which are labeled with carboxyfluorescein succinimidyl ester (CFSE).

c: The proportion of eTregs among total FOXP3 ${ }^{+}$TILs

d: Proportions of FOXP3 ${ }^{+}$cells and Treg subpopulations in breast cancer, PBMCs, and LNBT.

Page 14/15 
Figure 2
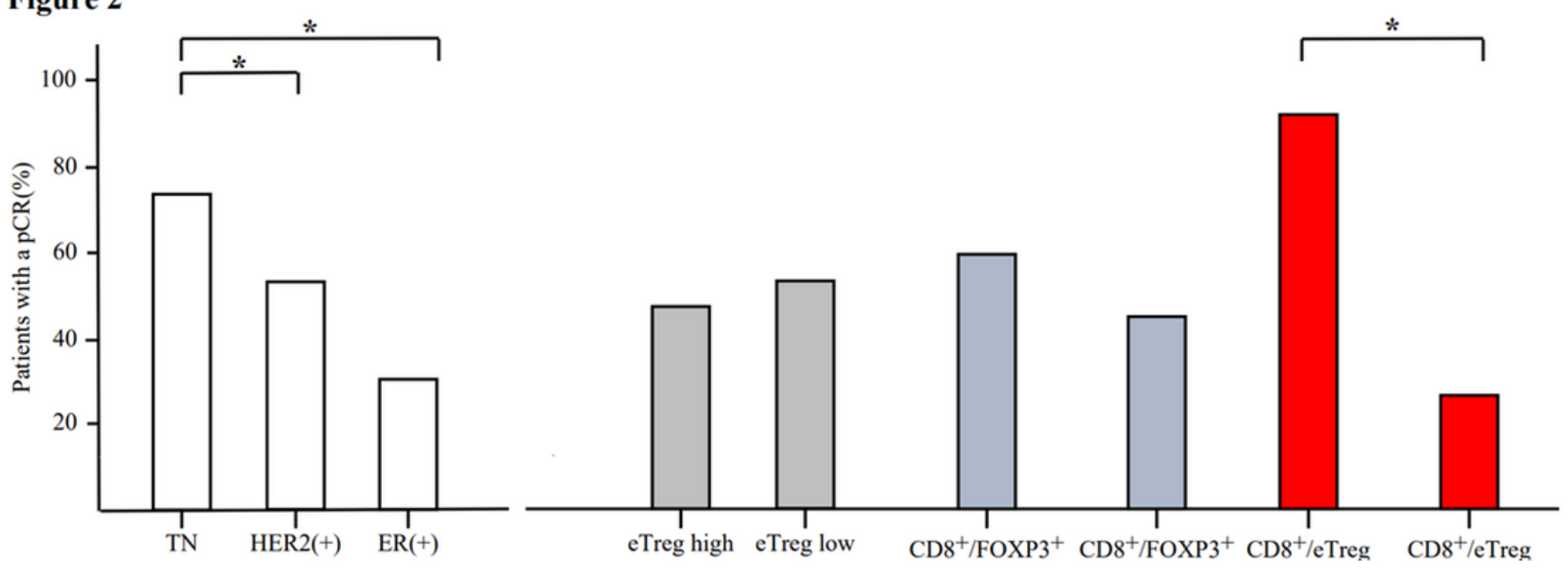

Figure 2

PCR rate of each breast cancer subtypes and variation of TIL subpopulations. pCR, pathological compete response. 